\title{
Prediction of mortality of APACHE-II and APACHE-III scores in patients admitted to the intensive care unit (ICU) for intoxication
}

\author{
E Banderas-Bravo ${ }^{1}$, MD Arias-Verdú ${ }^{*}$, I Macias-Guarasa ${ }^{1}$, E Aguilar-Alonso² ${ }^{2}$ E Castillo-Lorente ${ }^{3}$, R Rivera-Fernández ${ }^{1}$, \\ G Quesada- Garcia'
}

From ESICM LIVES 2015

Berlin, Germany. 3-7 October 2015

\section{Objective}

To study the characteristics and mortality in intoxicated patients admitted to the ICU, and prediction of mortality for usual prognostic indexes.

\section{Methods}

A multicenter study conducted from 2008 to 2013 in three Spanish hospitals. We collected clinical and demographics data. The differences between observed-to-predicted mortality were analyzed whith the Hosmer-Lemeshow test. Discrimination was evaluated by área under the ROC curve. $\mathrm{P}<0.005$ was statistically significant.

\section{Results}

We studied 119 patients. Mean age: $44.42 \pm 13.85$ years. Drug intoxication in $77.3 \%$ of patients, alcohol in $16.8 \%$ and caustic $9.2 \% .78 .3 \%$ was a suicide attempt. The mean Glasgow Coma Scale in $72.5 \%$ were $<8$ points. $69.7 \%$ required mechanical ventilation. The mortality of patients hospitalized for caustic ingestion was $54.5 \%$ and $1.9 \%$ in the rest $(\mathrm{p}<0.001)$.

The multivariate analysis showed that with SAPS-3 equal gravity (OR: $1.19(1.02-1.39)$ ) the mortality of patients who ingested caustics was higher (OR: 560.34 (11.64-26.973.83)) than the rest.

The mortality predicted by SAPS- 3 (general equation) was $26.98 \%$ and the observed $6.7 \%$, Hosmer-Lemeshow $(\mathrm{H}=36.47)(\mathrm{p}<0.001)$. With the APACHE-II and APACHE-III scores no statistically significant differences were observed, being the predicted mortality for APACHE-II of 7.57\% (Hosmer-Lemeshow Test: $\mathrm{H}=6.96$, no difference is) and for APACHE-III of 8.15\% (HosmerLemeshow Test: $\mathrm{C}=3.51$, not discrepancies).

\section{Conclusions}

APACHE-II and APACHE-III scores may adequately predict the probability of death in intoxicated patients, in contradistinction to the SAPS-3 score that overestimates the mortality. The ICU admission by intoxication is rare. A high percentage of patients have decreased level of consciousness and required mechanical ventilation. The mortality of patients hospitalized for caustic ingestion is higher to the rest.

\section{Authors' details}

${ }^{1}$ Hospital Regional Universitario Carlos Haya, Intensive Care Unit, Málaga, Spain. ${ }^{2}$ Hospital Infanta Margarita Cabra, Intensive Care Unit, Córdoba, Spain. ${ }^{3}$ Hospital Neurotraumatologico, Intensive Care Unit, Jaen, Spain.

Published: 1 October 2015

doi:10.1186/2197-425X-3-S1-A342

Cite this article as: Banderas-Bravo et al.: Prediction of mortality of APACHE-II and APACHE-III scores in patients admitted to the intensive care unit (ICU) for intoxication. Intensive Care Medicine Experimental 2015 3(Suppl 1):A342. 